\title{
FOURIER-TYPE ESTIMATION OF THE POWER GARCH MODEL WITH STABLE-PARETIAN INNOVATIONS ${ }^{1}$
}

\author{
C. FrancQ, S.G. Meintanis \\ CREST and University Lille 3, Villeneuve d'Ascq, FRANCE \\ e-mail: christian.francq@univ-lille3.fr \\ Department of Economics, National and Kapodistrian University of Athens \\ Athens, GREECE \\ and Centre for Business Mathematics and Informatics, North-West University \\ Potchefstroom, SOUTH AFRICA \\ e-mail: simosmei@econ.uoa.gr
}

\begin{abstract}
We consider the estimation of general power GARCH models with stableParetian innovations. Exploiting the simple structure of the conditional characteristic function of the observations driven by these models, we propose minimum distance estimation based on the empirical characteristic function of corresponding residuals. Consistency of the estimators is proved, and we obtain a singular asymptotic distribution which is concentrated on a hyperplane.
\end{abstract}

\section{Introduction}

Consider observations $y_{t}$ from the so-called power GARCH model defined by

$$
\left\{\begin{array}{l}
y_{t}=c_{t} \varepsilon_{t} \\
c_{t}^{\rho}=\mu+\sum_{j=1}^{p} b_{j}\left|y_{t-j}\right|^{\rho}+\sum_{j=1}^{q} \gamma_{j} c_{t-j}^{\rho}, \quad \forall t=1,2, \ldots,
\end{array}\right.
$$

where $\left\{\varepsilon_{t}\right\}$ is a sequence of $i . i . d$. random variables (with location zero and unit scale) independent of $\left\{c_{t}\right\}$, and $\rho, \mu,\left\{b_{j}\right\}_{j=1}^{p}$, and $\left\{\gamma_{j}\right\}_{j=1}^{q}$ denote unknown parameters. If the innovations $\left\{\varepsilon_{t}\right\}$ are standard normal and the power parameter $\rho$ is set equal to two we obtain the classical Gaussian GARCH model. From the time of Mandelbrot (1963) and Fama (1965) however there is strong evidence that the distribution of financial returns could be heavy-tailed and possibly asymmetric, and many authors advocated the use of the stable-Paretian (SP) distribution instead of the normal distribution in financial modelling. For more recent evidence of stable-Paretian behavior of financial assets the reader is referred to the papers of Mittnik and Rachev (1993), Koutrouvelis and Meintanis (1999), Liu and Brorsen (1995a), Paolella (2001), Tsionas (2002), Akgül and Sayyan (2008), Tavares et al. (2008), Curto et al. (2009), and Xu et al. (2011), and the volumes by Adler et al. (1998), Rachev and Mittnik (2000), Rachev (2003) and Nolan, 2012).

\footnotetext{
${ }^{1}$ This research was partially supported by Grant No. 11699 of the Special Account for Research Grants of the National and Kapodistrian University of Athens, and by the Agence Nationale de la Recherche (ANR) and the Economic and Social Research Council (ESRC) through the ORA Program (Project PRAM ANR-10-ORAR-008-01)
} 
Therefore one of the popular generalizations of model (1.1) is to assume that $\left\{\varepsilon_{t}\right\}$ follow a stable-Paretian distribution. We shall call this model SP power GARCH (SPPGARCH) model. The most convenient way to introduce SP distributions is by means of their characteristic function $(\mathrm{CF})$. Specifically if we assume that $\varepsilon_{t}$ are zero-location $\mathrm{SP}$ random variables with unit scale, then their $\mathrm{CF}$ is given by

$$
\begin{aligned}
\varphi(u, \boldsymbol{\lambda}) & =e^{-|u|^{\alpha}\{1-\mathrm{i} \beta \operatorname{sgn}(u) \tan (\pi \alpha / 2)\}}, \quad \alpha \neq 1, \\
& =e^{-|u|\left\{1+\mathrm{i} \beta \frac{2}{\pi} \operatorname{sgn}(u) \log |u|\right\}}, \quad \alpha=1,
\end{aligned}
$$

where $\boldsymbol{\lambda}=(\alpha, \beta)^{\prime}$ is the vector of the SP parameter such that $0<\alpha \leq 2$ and $-1 \leq \beta \leq$ 1. We also used the notation $\operatorname{sgn}(u)=1, u>0, \operatorname{sgn}(0)=0$, and $\operatorname{sgn}(u)=-1, u<0$. Note that $\alpha$ is a shape parameter often referred to as the 'tail index' and that the SP law reduces to the Gaussian distribution at $\alpha=2$. On the other hand $\beta$ measures skewness, and if $\beta=0$ the corresponding SP law is symmetric. Besides the normal law, well known particular cases are the Cauchy distribution for $(\alpha, \beta)=(1,0)$ and the Lévy distribution which corresponds to $(\alpha, \beta)=(1 / 2,1)$. Several authors (see for instance Mittnik et al. 1999, Liu and Brorsen 1995b, and Bonato 2009) proposed maximum likelihood estimation of the SP-PGARCH model. However since the density of the SP law is generally not available in closed form various approximations are needed, and therefore likelihood methods may be characterized as computationally demanding.

In the next section we capitalize on the simplicity of eq. (1.2) and suggest an estimation procedure based on a comparison between the empirical $\mathrm{CF}$ (ECF) of the PGARCH residuals and the (theoretical) CF of the SP distribution. With the ECFbased estimators that have been proposed in the literature, the ECF depends on the observations and the CF depends on the unknown parameter. Our estimator is different because the ECF not only depends on the observations but also on the GARCH parameter, whereas the $\mathrm{CF}$ depends on the SP parameter.

\section{CF estimation of the SP-PGARCH model}

Consider the SP-PGARCH model whereby the observations $y_{t},(t=1, \ldots, T)$, are driven by equation (1.1) and the innovations $\varepsilon_{t}$ have CF given by (1.2). We assume the standard positivity conditions $\mu>0,\left\{b_{j} \geq 0,1 \leq j \leq p\right\}$ and $\left\{\gamma_{j} \geq 0,1 \leq j \leq q\right\}$.

Denote by $\boldsymbol{\theta}=\left(\rho, \mu, b_{1}, \ldots, b_{p}, \gamma_{1}, \ldots, \gamma_{q}\right)^{\prime}$ the PGARCH parameter and by $\boldsymbol{\lambda}=$ $(\alpha, \beta)^{\prime}$ the SP parameter. We suggest to estimate the parameter $\boldsymbol{\vartheta}=\left(\boldsymbol{\theta}^{\prime}, \boldsymbol{\lambda}^{\prime}\right)^{\prime}$, by minimum distance between the $\mathrm{CF}$ and a suitable empirical counterpart. Specifically, given the observations $\left(y_{1}, \ldots, y_{T}\right)$ and fixed initial values $\left(y_{0}, \ldots, y_{1-p}\right)$ and $\left(\widetilde{c}_{0}, \ldots, \widetilde{c}_{1-q}\right)$, the estimation method is defined as

$$
\widehat{\boldsymbol{\vartheta}}_{T}=\arg \min _{\boldsymbol{\vartheta} \in \Xi} \widetilde{\Delta}_{T}(\boldsymbol{\vartheta}),
$$

where $\Xi$ denotes the parameter space and

$$
\widetilde{\Delta}_{T}(\boldsymbol{\vartheta})=\int_{-\infty}^{\infty}\left|\widetilde{\varphi}_{T}(u, \boldsymbol{\theta})-\varphi(u, \boldsymbol{\lambda})\right|^{2} W(u) d u,
$$


with $W(\cdot)$ a nonnegative weight function. In $(2.2) \widetilde{\varphi}_{T}(u, \boldsymbol{\theta}):=\varphi_{T}\left\{u ; \widetilde{\varepsilon}_{1}(\boldsymbol{\theta}), \ldots, \widetilde{\varepsilon}_{T}(\boldsymbol{\theta})\right\}$ is the empirical $\mathrm{CF}$ (ECF) defined by

$$
\varphi_{T}\left(u ; x_{1}, \ldots, x_{T}\right)=\frac{1}{T} \sum_{t=1}^{T} e^{\mathrm{i} u x_{t}},
$$

and computed from the residuals $\widetilde{\varepsilon}_{t}(\boldsymbol{\theta})=y_{t} / \widetilde{c}_{t}(\boldsymbol{\theta})$, with $\widetilde{c}_{t}(\boldsymbol{\theta})$ being recursively defined for $t \geq 1$, by

$$
\widetilde{c}_{t}^{\rho}(\boldsymbol{\theta})=\mu+\sum_{j=1}^{p} b_{j}\left|y_{t-j}\right|^{\rho}+\sum_{j=1}^{q} \gamma_{j} \widetilde{c}_{t-j}^{\rho}(\boldsymbol{\theta}) .
$$

Note that the introduction of the weight function $W(\cdot)$ in $(2.2)$ is necessary in order to neutralize the periodic components in the $\operatorname{ECF} \widetilde{\varphi}_{T}(u, \boldsymbol{\theta})$ and thus render the corresponding integral finite.

ECF-based estimation methods date back to Heathcote (1977), Bryant and Paulson (1979) and Thornton and Paulson (1997), for i.i.d. data. There is also work on ECFbased estimation for dependent data. The reader is referred to Feuerverger (1990), Singleton (2001), Knight and Yu (2002), Knight et al. (2002), Yu (2004), Carrasco et al. (2007), Kotchoni (2012), and references therein.

Note that the ECF, $\widetilde{\varphi}_{T}(u, \boldsymbol{\theta})$, involves the PGARCH parameter and the CF, $\varphi(u, \boldsymbol{\lambda})$, involves the SP parameter. In the existing literature, the unknown parameter is only involved in the CF, the ECF being computed directly from the observations $y_{1}, \ldots, y_{T}$. A major difference with the estimator defined by (2.1)-(2.4) and the estimators considered in the above-mentioned references is that we consider an ECF of residuals instead of an ECF of observations. The reason why we can not use the standard approach in our framework is that, for a PGARCH model, there exists no closed form for the $\mathrm{CF}$ of a vector of the form $\left(y_{t}, \ldots, y_{t-h}\right), h \geq 0$.

\section{$3 \quad$ Asymptotic properties}

Now consider the asymptotic properties of the estimator (2.1) of the parameter of the $\mathrm{SP}-\mathrm{PGARCH}(p, q)$ model (1.1)-(1.2). Recall that the parameter vector is decomposed as $\boldsymbol{\vartheta}=\left(\boldsymbol{\theta}^{\prime}, \boldsymbol{\lambda}^{\prime}\right)^{\prime}$ with $\boldsymbol{\theta}=\left(\rho, \mu, b_{1}, \ldots, b_{p}, \gamma_{1}, \ldots, \gamma_{q}\right)^{\prime} \in \Theta$ and $\boldsymbol{\lambda}=(\alpha, \beta)^{\prime} \in \Lambda$. The true parameter value is denoted by $\boldsymbol{\vartheta}_{0}=\left(\boldsymbol{\theta}_{0}^{\prime}, \boldsymbol{\lambda}_{0}^{\prime}\right)^{\prime}$ with $\boldsymbol{\theta}_{0}^{\prime}=\left(\rho_{0}, \mu_{0}, b_{01}, \ldots, b_{0 p}, \gamma_{01}, \ldots, \gamma_{0 q}\right)$ and $\boldsymbol{\lambda}_{0}^{\prime}=\left(\alpha_{0}, \beta_{0}\right)$. Following the seminal paper of Bougerol and Picard (1992), it is easy to see that the necessary and sufficient condition for strict stationarity of (1.1) takes the form $\gamma\left(\boldsymbol{\theta}_{0}\right)<0$, where $\gamma\left(\boldsymbol{\theta}_{0}\right)$ is the top-Lyapounov exponent of the model, as defined in Appendix A of Hamadeh and Zakoïan (2011).

In addition to this strict stationarity condition and to standard identifiability conditions, the strong consistency requires the following assumptions:

$$
\begin{aligned}
\boldsymbol{\vartheta}_{0} \in \Xi:= & \Theta \times \Lambda \text { where } \Theta \text { is a compact subset of }(0, \infty)^{2} \times[0, \infty)^{p+q} \\
& \text { and } \Lambda \text { is a compact subset of }(1,2) \times[-1,1]
\end{aligned}
$$


and

$$
W(\cdot) \text { is strictly positive over } \mathbb{R} \backslash\{0\} \text {, with } \int|u|^{j} W(u) d u<\infty
$$

for $j=0$ and $j=1$. Assumption (3.1) imposes standard positivity constraints on the PGARCH coefficients. It also puts restrictions on the value of the tail index $\alpha_{0}$. DuMouchel (1983) showed that, in the case of a sample of stable distribution, the asymptotic distribution of the MLE is not standard when $\alpha_{0}=2$. Note also that when $\alpha_{0}=2$, the coefficient $\beta_{0}$ is not identifiable. We impose $\alpha_{0}>1$ because we need $E\left|\varepsilon_{t}\right|<\infty$.

It is also assumed that $\sum_{j=1}^{q} \gamma_{j}<1$ for all $\boldsymbol{\theta} \in \Theta$. A stationary sequence $\left\{c_{t}(\boldsymbol{\theta})\right\}$ can then be defined such that $\left|c_{t}(\boldsymbol{\theta})-\widetilde{c}_{t}(\boldsymbol{\theta})\right| \rightarrow 0$ almost surely as $t \rightarrow \infty$. To show the identifiability of the conditional characteristic function, the following assumption is also needed:

$$
E \sup _{\boldsymbol{\theta} \in \Theta}\left\{\frac{c_{1}\left(\boldsymbol{\theta}_{0}\right)}{c_{1}(\boldsymbol{\theta})}\right\}^{10}<\infty
$$

For an $\operatorname{ARCH}(p)$ model, we have

$$
\frac{c_{t}\left(\boldsymbol{\theta}_{0}\right)}{c_{t}(\boldsymbol{\theta})}=\left(\frac{\mu_{0}+\sum_{j=1}^{p} b_{0 j} y_{t-j}^{\rho}}{\mu+\sum_{j=1}^{p} b_{j} y_{t-j}^{\rho}}\right)^{1 / \rho} \leq\left(\frac{\mu_{0}}{\mu}+\sum_{j=1}^{p} \frac{b_{0 j}}{b_{j}}\right)^{1 / \rho} .
$$

Therefore, in the ARCH case, (3.2) is satisfied when $\inf _{\boldsymbol{\theta} \in \Theta} \min b_{j}>0$. In the general case, it can be shown that (3.2) is satisfied when $\Theta$ is sufficiently small (see (5.15) and (5.16) in Hamadeh and Zakoïan (2011), referred to as HZ hereafter).

Under the previous assumptions, any sequence $\left(\widehat{\boldsymbol{\vartheta}}_{T}\right)$ of CF estimators satisfying (2.1) is strongly consistent:

$$
\widehat{\boldsymbol{\vartheta}}_{T} \rightarrow \boldsymbol{\vartheta}_{0} \quad \text { almost surely, as } T \rightarrow \infty \text {. }
$$

To obtain the asymptotic normality, it is obviously necessary to assume that $\boldsymbol{\vartheta}_{0}$ does not stand at the boundary of the parameter space $\Xi$. We also need to introduce few additional notations. Let

$$
g_{t}(u, \boldsymbol{\vartheta})=e^{\mathrm{i} u \varepsilon_{t}(\boldsymbol{\theta})}-\varphi(u, \boldsymbol{\lambda})
$$

and the vector of dimension $d=p+q+3$

$$
\boldsymbol{\Upsilon}_{t}=\int_{-\infty}^{\infty} \operatorname{Re}\left(\overline{g_{t}\left(u, \boldsymbol{\vartheta}_{0}\right)} E \frac{\partial g_{1}\left(u, \boldsymbol{\vartheta}_{0}\right)}{\partial \boldsymbol{\vartheta}}\right) W(u) d u
$$

It is then possible to show the existence of the matrices $\mathbf{V}=\operatorname{Var} \boldsymbol{\Upsilon}_{1}$ and

$$
\mathbf{G}=\int_{-\infty}^{\infty} \operatorname{Re}\left(E \frac{\partial \overline{g_{1}\left(u, \boldsymbol{\vartheta}_{0}\right)}}{\partial \boldsymbol{\vartheta}} E \frac{\partial g_{1}\left(u, \boldsymbol{\vartheta}_{0}\right)}{\partial \boldsymbol{\vartheta}^{\prime}}\right) W(u) d u .
$$

The matrices $\mathbf{V}$ and $\mathbf{G}$ are actually singular, and one can show that

$\mathbf{G} \sqrt{T}\left(\widehat{\boldsymbol{\vartheta}}_{T}-\boldsymbol{\vartheta}_{0}\right)$ converges in law to the $\mathcal{N}(\mathbf{0}, \mathbf{V})$ distribution as $T \rightarrow \infty$. 
By showing that $\mathrm{G}=\boldsymbol{A} \boldsymbol{B} \boldsymbol{A}^{\prime}$ where

$$
\boldsymbol{A}=\left(\begin{array}{cc}
E \frac{1}{c_{1}} \frac{\partial c_{1}\left(\boldsymbol{\theta}_{0}\right)}{\partial \boldsymbol{\theta}} & \mathbf{0} \\
\mathbf{0} & \boldsymbol{I}_{2}
\end{array}\right)
$$

has full rank 3 and $\boldsymbol{B}$ is an invertible $3 \times 3$ matrix, we conclude that $\sqrt{T} \boldsymbol{A}^{\prime}\left(\widehat{\boldsymbol{\vartheta}}_{T}-\boldsymbol{\vartheta}_{0}\right)$ converges in law to the $\mathcal{N}(\mathbf{0}, \boldsymbol{\Sigma})$ distribution, for some matrix $\boldsymbol{\Sigma}$. When $W(\cdot)$ is even the latter matrix is of the form

$$
\Sigma=\left(\begin{array}{ll}
0 & 0 \\
0 & S
\end{array}\right)
$$

It follows that the asymptotic distribution of $\sqrt{T}\left(\widehat{\boldsymbol{\theta}}_{T}-\boldsymbol{\theta}_{0}\right)$ is concentrated on the line

$$
\Delta_{c}=\left\{\boldsymbol{x} \in \mathbb{R}^{p+q+2}: \boldsymbol{x}^{\prime} E \frac{1}{c_{t}\left(\boldsymbol{\theta}_{0}\right)} \frac{\partial c_{t}\left(\boldsymbol{\theta}_{0}\right)}{\partial \boldsymbol{\theta}}=c\right\}
$$

for some constant $c$.

\section{A numerical illustration}

The aim of the simulation experiment presented in this section is to illustrate that the ECF is consistent, but has a non standard asymptotic distribution concentrated on a line, as stated in the previous section. We thus consider the following very simple version of the SP-PGARCH model

$$
y_{t}=c_{t} \varepsilon_{t}, \quad c_{t}^{2}=\mu_{0}+b_{0} y_{t-1}^{2}
$$

where $\boldsymbol{\vartheta}_{0}=(0.5,0.2,1.6,0)$, i.e., we have a SP-PARCH(1) model with symmetric SP innovations and tail index equal to 1.6. Moreover we assume that the value of $\rho$ is known to be equal to 2. We estimated the four parameters by ECF over $N=1,000$ independent simulations of length $T=20,000$ of the process. As expected, because the sample size is largen for each parameter, the estimated values were very close to the true value. Figure 1 displays the scatter plot of the 1,000 values of $\left(\hat{\mu}-\mu_{0}, \hat{b}-b_{0}\right)$. In accordance with the previous section, the points are concentrated along the red line, carried by the vector

$$
E \frac{1}{c_{t}\left(\boldsymbol{\theta}_{0}\right)} \frac{\partial c_{t}\left(\boldsymbol{\theta}_{0}\right)}{\partial \boldsymbol{\theta}} ?^{2}
$$

For comparison, we plotted the linear regression of $\hat{b}-b_{0}$ on $\hat{\mu}-\mu_{0}$ as a dotted line. This line is almost confused with the full red line.

\footnotetext{
${ }^{2}$ Because there exists no explicit form for this vector, it has been evaluated on the basis of a simulation of length 50,000.
} 


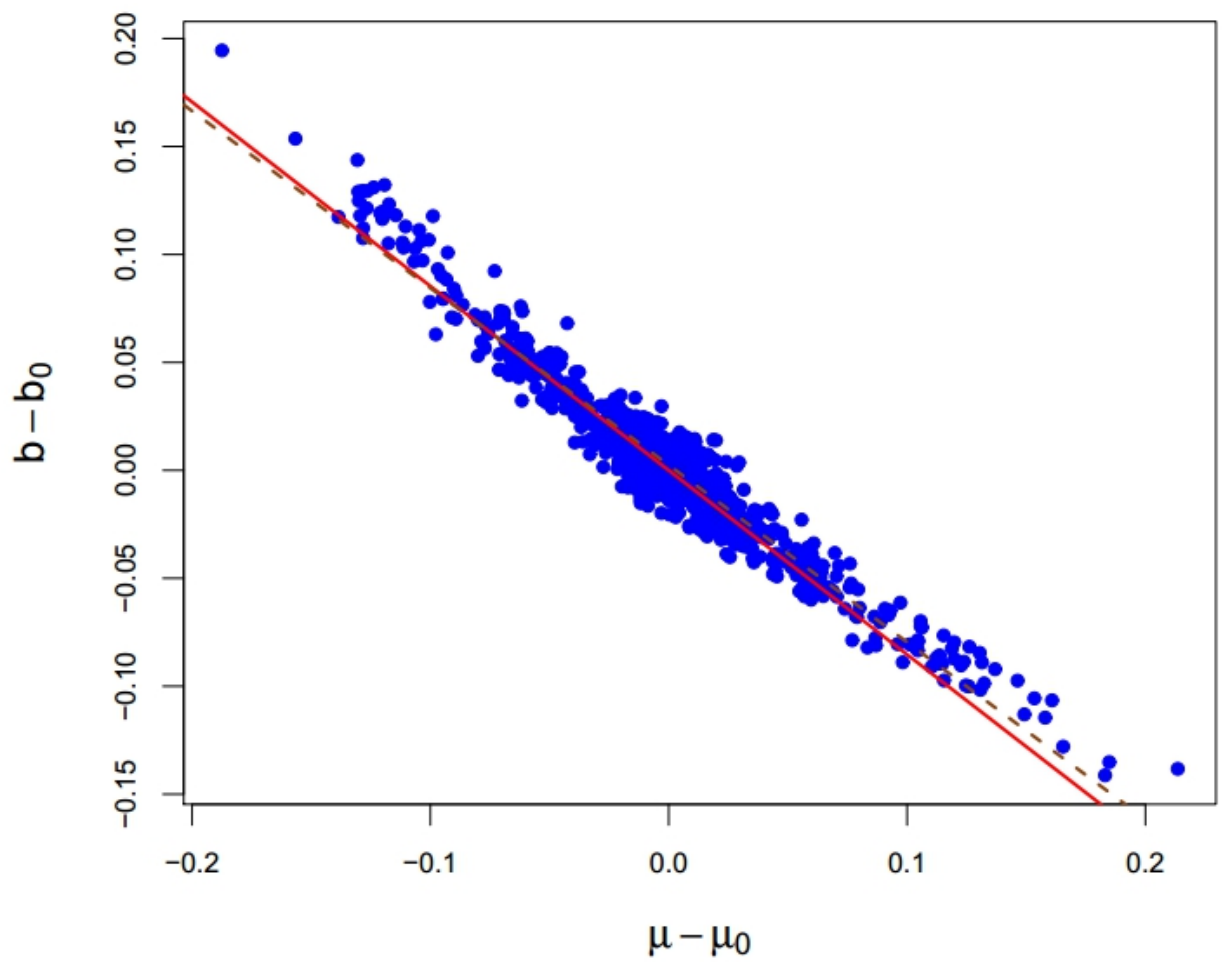

Figure 1: Empirical distribution of the ECF estimator over 1,000 independent simulations of length $T=20,000$ of the SP-PARCH(1) model (4.1). The red line of the scatter plot corresponds to the direction of the vector $E c^{-1} \partial c_{t}\left(\boldsymbol{\theta}_{0}\right) / \partial \boldsymbol{\theta}$. 


\section{References}

[1] Adler R.J., Feldman R.E., Taqqu M.S. (1998). A Practical Guide to Heavy Tails. Statistical Techniques and Applications. Birkhäuser, Boston.

[2] Akgül I., Sayyan H. (2008). Modelling and forecasting long memory in exchange rate volatility vs. stable and integrated GARCH models. Appl. Financ. Econom.. Vol. 18, pp. 463-482.

[3] Bryant J.L. and Paulson A.S. (1979). Some comments on characteristic function based estimators. Sankhyā. Vol. 41, pp. 109-116.

[4] Bonato M. (2009). Modeling fat tails in stock returns: a multivariate stable-GARCH approach. Electronic copy available at http://ssrn.com/abstract $=1015477$.

[5] Bougerol P., Picard N. (1992). Stationarity of GARCH processes and of some nonnegative time series. J. Econometr.. Vol. 52, pp. 115-127.

[6] Curto J.D., Pinto J.C., Tavares G.N. (2009). Modelling stock markets' volatility using GARCH models with Normal, Student's $t$ and stable Paretian distribution. Statist. Papers. Vol. 50, pp. 311-321.

[7] DuMouchel W.H. (1983). Estimating the stable index $\alpha$ in order to measure tail thickness: A critique. Ann. Statist.. Vol. 11, pp. 1019-1031.

[8] Fama E. (1965). The behavior of stock market prices. J. Business. Vol. 38,pp. 34105.

[9] Feuerverger A. (1990). An efficiency result for the empirical characteristic function in stationery time-series models. Canad. J. Statist.. Vol. 18, pp. 155-161.

[10] Hamadeh T., Zakoïan J.M. (2011). Asymptotic properties of LS and QML estimators for a class of nonlinear GARCH processes. J. Statist. Plann. Inference. Vol. 141, pp. 488-507.

[11] Heathcote C.R. (1977). The integrated squared error estimation of parameters. Biometrika. Vol. 64, pp. 255-264.

[12] Knight J.L., Yu J. (2002). The empirical characteristic function in time series estimation. Econometric Theory. Vol. 18, pp. 691-721.

[13] Kotchoni R. (2012). Applications of the characteristic function-based continuum GMM in finance. Comput. Statist. Dat. Anal. . Vol. 56, pp. 3599-3622.

[14] Koutrouvelis I.A., Meintanis S.G. (1999). Testing for stability based on the empirical characteristic function with applications to financial data. J. Statist. Comput. Simul.. Vol. 64, pp. 275-300. 
[15] Liu S.M., Brorsen B.W. (1995a). GARCH-stable as a model for future price movements. Rev. Quantitat. Financ. Account.. Vol. 5, pp. 155-167.

[16] Liu S.M., Brorsen B.W. (1995b). Maximum likelihood estimation of a GARCHstable model. J. Appl. Econometr.. Vol. 10, pp. 273-285.

[17] Mandelbrot B. (1963). The variation of certain speculative prices. J. Business. Vol. 36, pp. 394-419.

[18] Mittnik S., Rachev S.T. (1993). Modelling asset returns with alternative stable models. Econometr. Rev.. Vol. 12, pp. 261-330.

[19] Mittnik S., Rachev S.T., Doganoglu T, Chenyao D. (1999). Maximum likelihood estimation of stable Paretian models. Math. Comput. Model.. Vol. 29, pp. 275293.

[20] Nolan J.P. (2012). Stable Distributions - Models for Heavy Tailed Data. Birkhauser, Boston.

[21] Paolella M.S. (2001). Testing the stable Paretian assumption. Math. Comput. Model. . Vol. 34, pp. 1095-1112.

[22] Rachev S. (2003). Handbook of Heavy Tailed Distributions in Finance. Elsevier/North-Holland, Amsterdam.

[23] Rachev S., Mittnik S. (2000). Stable Paretian Models in Finance. Wiley, New York.

[24] Singleton K.J. (2001). Estimation of affine asset pricing models using the empirical characteristic function. J. Econometr.. Vol. 102, pp. 111-141.

[25] Tavares A.B., Curto J.D., Tavares G.N. (2008). Modelling heavy tails and asymmetry using ARCH-type models with stable Paretian distribution. Nonlinear Dyn.. Vol. 51, pp. 231-243.

[26] Thornton J.C. and Paulson A.S. (1977). Asymptotic distribution of characteristic function-based estimators for the stable laws. Sankhyā. Vol. 39, pp. 341-354.

[27] Tsionas E. (2002). Likelihood-based comparison of stable Paretian and competing models: Evidence from daily exchange rates. J. Statist. Comput. Simul. . Vol. 72, pp. 341-353.

[28] Xu W., Wu C., Dong Y., Xiao W. (2011). Modeling Chinese stock returns with stable distribution. Math. Comput. Model.. Vol. 54, pp. 610-617.

[29] Yu J. (2004). Empirical characteristic function estimation and its applications. Econometric Rev.. Vol. 23,pp. 93-123. 